appendix, with its samples of 'physiological drawings': teaching children how their bodies function might better be undertaken when they are not ill and away from home. I found no reference by the author to an out-patient appointment system, such as has proved so helpful in this country.

The subtitle of this readable attractive book is: 'A Guide for the Professional Team'. The author gives well-considered and helpful guidance, not only for formal teams, but for all those individuals in the medical, auxiliary and administrative services, who work for and think about children in hospitals.

\section{Grundriss der Pathologischen Embryologie des Menschen.} By ANDrej P. Dyban. (Pp. viii - 260;83 figures 11 tables. DM. 47,10.) Jena: Gustav Fischer. 1962.

Recent concern with drug-induced abnormalities in foetal development makes this monograph timely, for it serves to draw attention to the wide range of conditions -some without any apparent deleterious effect upon the mother-under which disturbances of embryogenesis can take place.

An early chapter deals with natural abortion, which in England, Germany and the United States is believed to involve the death of some $10^{\circ}$ of all fertilized ova, and with its frequency in the successive weeks of pregnancy. The large majority take place during the first trimester, and male foetuses are lost some four times more commonly than female. One long chapter surveys the possible teratogenic actions of such adverse factors as temperature changes, hypoxia, ionizing radiation, toxic chemicals, undernutrition, hormonal influences and infections. Much of the material incorporated has been obtained from animal experiments in which the action of the teratogenic agent has been pressed more severely than it is ever likely to operate in man. Indeed, references to human instances of their action are for the most part incidental.

The morphological features of abortion are dealt with in a long chapter which contains many illustrations obtained from human material. The peculiar hazards found at two critical periods are emphasized: the former during the first few days of gestation and the second between the third and the sixth weeks. Much of the later part of the monograph is concerned with the vital staining, histochemistry and hormonal activities of the placenta.

The book is a translation into German of a Russian monograph published at Lvov in 1959. It contains nearly a thousand references of which about one-third are to articles in Russian; the rest are widely distributed in the publications of most western countries. The format is fair, and the illustrations - many from borrowed sources-are well reproduced. The monograph could be useful to those interested in the problems of foetal malformations and abortion.

Dunham's Premature Infants. 3rd ed. By William A. Silverman. (Pp. xii +578 ; illustrated. \$15.00.) New York: Hoeber; London: Hamilton. 1961.

The third edition of Dunham's Premature Infants is necessarily a larger volume than the second edition of 1955. It retains the same basic organization of three main sections. Part 1 deals with general considerations, Part 2 with clinical aspects and Part 3 with public health. Part 2 has been further divided into two sections. The first of these contains seven chapters dealing with the care of premature infants, and the second contains 11 chapters on abnormal conditions, their signs, symptoms and treatment.

It is this clinical part that has been swelled by the inclusion of much of the new information that has been gained over the past few years. There is a long chapter on retrolental fibroplasia, a new chapter on the infants of diabetic mothers and a wealth of detail about respiratory distress syndrome. It is disappointing that the latter does not include more details regarding the treatment of this condition but it is the policy of the author not to be dogmatic about controversial subjects.

A number of appendices giving data on normal values and techniques completes the book.

Despite the increase in the amount of information given, the book remains very readable and is presented in a convenient size for handling. Clear sub-headings, good print and excellent charts and photographs make for easy reference. No aspect of health or disease is left unconsidered and controversial points of view are clearly represented. The succinct script allows a rapid appraisal of the diagnosis and treatment of abnormal states and the daily problems of nursery management. The practice and experience of the Babies' Hospital, New York, is quoted wherever controversy does not allow an unequivocal recommendation and serves as a guide for those who require it. The conciseness at times gives rise to a brevity amounting almost to incompleteness, but if the text is wanting this is made good by the excellent bibliography (about 1,500 references in all).

Reference to other parts of the book by chapter rather than page is annoying and the index occasionally inadequate, but these criticisms do not detract from the pleasure in reading this very informative text. 